\title{
A TWO-SIDED ABSORPTION PROBLEM
}

\section{HAROLD WIDOM}

1. In this note we consider a possibly asymmetric Cauchy process $X(t), t \geqq 0$, with transition probability densities

$$
p(x, y, t)=\frac{d}{d y} \operatorname{Pr}\{X(t) \leqq y \mid X(0)=x\},=\frac{1}{\pi} \frac{t}{t^{2}+(x-y-a t)^{2}} .
$$

Assuming as we may that the paths are right continuous, we denote by $T_{x}$ the first passage time to the exterior of the open unit interval given that the process starts at $x$;

$$
T_{x}=\min \{t:|X(t)| \geqq 1\} .
$$

We shall obtain explicit formulas for the moments of $T_{x}$, and also for the distribution of the place of first passage $X\left(T_{x}\right)$. In the case of the symmetric Cauchy process these were obtained by Kac and Pollard [2] and Spitzer [4] respectively.

2. We set

$$
k(x, y, t)=\frac{d}{d y} \operatorname{Pr}\left\{\max _{\tau \leqq t}|X(\tau)|<1 \text { and } X(t) \leqq y \mid X(0)=x\right\} .
$$

Then by the extended Markov property we have

$$
\begin{aligned}
p(x, y, t)= & k(x, y, t) \\
& +\iint_{|z| \geq 1, \tau \leq t} p(z, y, t-\tau) d_{z, \tau} \operatorname{Pr}\left\{X\left(T_{x}\right) \leqq z, T_{x} \leqq \tau\right\} .
\end{aligned}
$$

Set

$$
K(x, y)=\int_{0}^{\infty} k(x, y, t) d t .
$$

It follows from the inequalities

$$
\begin{aligned}
k(x, y, t) & \leqq p(x, y, t), \\
k(x, y, t) & \leqq \int_{-1}^{1} p\left(x, z, \frac{t}{2}\right) p\left(z, y, \frac{t}{2}\right) d z,
\end{aligned}
$$

Presented to the Society, January 23, 1961 under the title The 2-sided absorption problem for asymmetric Cauchy processes; received by the editors December 2, 1960. 
which, of course, $k$ satisfies, that we have an estimate

$$
K(x, y) \leqq A \log \left(1+|x-y|^{-1}\right) .
$$

If we multiply (1) by $e^{i \xi y}$ and integrate with respect to $y$ over $(-\infty, \infty)$ and then with respect to $t$ over $(0, \infty)$ we obtain

$$
\begin{aligned}
\frac{e^{i \xi x}}{|\xi|+i a \xi}= & \int_{-1}^{1} e^{i \xi y} K(x, y) d y \\
& +\frac{1}{|\xi|+i a \xi} \int_{|z| \geq 1} e^{i \xi y} d \operatorname{Pr}\left\{X\left(T_{x}\right) \leqq z\right\} .
\end{aligned}
$$

We rewrite this as

(4) $\frac{e^{i \xi x}}{\xi}=(\operatorname{sgn} \xi+i a) \int_{-1}^{1} e^{i \xi y} K(x, y) d y+\int_{|z| \geq 1} \frac{e^{i \xi z}}{\xi} d \operatorname{Pr}\left\{X\left(T_{x}\right) \leqq z\right\}$.

Now $\operatorname{sgn} \xi \int_{-1}^{1} e^{i \xi y} K(x, y) d y$ is the Fourier transform of the Hilbert transform of $K(x, y)$, i.e.

$$
\operatorname{sgn} \xi \int_{-1}^{1} e^{i \xi y} K(x, y) d y=\frac{i}{\pi} \int_{-\infty}^{\infty} e^{i \xi u} d u \int_{-1}^{1} \frac{K(x, y)}{y-u} d y .
$$

Therefore (4) gives easily

$$
\begin{aligned}
\frac{i}{2} \operatorname{sgn}(x-u)= & \frac{i}{\pi} \int_{-1}^{1} \frac{K(x, y)}{y-u} d y+i a K(x, u) \\
& +\frac{i}{2} \int_{|z| \geq 1} \operatorname{sgn}(z-u) d \operatorname{Pr}\left\{X\left(T_{x}\right) \leqq z\right\} .
\end{aligned}
$$

Introducing the unit step function

$$
\chi(y)= \begin{cases}0, & y<0, \\ 1, & y \geqq 0,\end{cases}
$$

the last equation may be written

(5) $\operatorname{Pr}\left\{X\left(T_{x}\right) \leqq u\right\}-\chi(u-x)=\frac{1}{\pi} \int_{-1}^{1} \frac{K(x, y)}{y-u} d y+a K(x, u)$.

This holds for all $u$ in $|u|>1$, and certainly almost all $u$ in $|u| \leqq 1$. In particular we obtain the singular integral equation

$$
\operatorname{Pr}\left\{X\left(T_{x}\right) \leqq-1\right\}-\chi(u-x)
$$

$$
=\frac{1}{\pi} \int_{-1}^{1} \frac{K(x, y)}{y-u} d y+a K(x, u), \quad|u| \leqq 1 .
$$


3. It is known how to solve equations such as (6). (See, for example, $[5, \S 4.4]$.) We shall reproduce the solution here since we shall need, in addition to the determination of $K$ from the left side of (6), the determination of the left side of (5). (One can of course substitute the computed $K$ into the right of (5), but it is even easier than that.) We state the relevant results as a lemma.

Denote by $2 \pi \beta$ that argument of $(a-i) /(a+i)$ which lies between 0 and $2 \pi$ and set

$$
\gamma(u)=|u+1|^{\beta}|u-1|^{1-\beta} .
$$

Then:

Assume $f \in L_{2}(-1,1)$ and

$$
\phi(u)=\frac{1}{\pi} \int_{-1}^{1} \frac{f(y)}{y-u} d y+a f(u) \quad \text { p.p.in }|u| \leqq 1 .
$$

If $\phi$ is defined by the right side of (7) for $|u|>1$ (considering $f$ to be 0 there), then

(8) $\phi(u)=-\frac{1}{\pi\left(a^{2}+1\right)^{1 / 2} \gamma(u)} \int_{-1}^{1} \frac{\phi(y) \gamma(y)}{|y-u|} d y+\frac{C \operatorname{sgn} u}{\gamma(u)}|u|>1$

for some constant $C$. Moreover, with the same $C$,

$$
\begin{aligned}
f(u)= & \frac{a}{a^{2}+1} \phi(u)-\frac{1}{\pi\left(a^{2}+1\right) \gamma(u)} \int_{-1}^{1} \frac{\phi(y) \gamma(y)}{y-u} d y \\
& -\frac{C}{\left(a^{2}+1\right)^{1 / 2} \gamma(u)} \quad \text { p.p. in }|u| \leqq 1 .
\end{aligned}
$$

Let us assume first that $f$, when extended to $|u|>1$ by being given the value 0 there, is twice continuously differentiable. Then the function

$$
F_{+}(w)=\frac{1}{2 \pi} \int_{-1}^{1} \frac{f(y)}{y-w} d y,
$$

which is analytic in $g w>0$, is continuous in $g w \geqq 0$ when defined for real $u$ by

$$
F_{+}(u)=\frac{i}{2} f(u)+\frac{1}{2 \pi} \int_{-1}^{1} \frac{f(y)}{y-u} d y,
$$

where of course the integral is now a principal value. Similarly the function 


$$
F_{-}(w)=\frac{1}{2 \pi} \int_{-1}^{1} \frac{f(y)}{y-w} d y
$$

is analytic in $g w<0$ and continuous in $g w \leqq 0$ when defined for real $u$ by

$$
F_{-}(u)=-\frac{i}{2} f(u)+\frac{1}{2 \pi} \int_{-1}^{1} \frac{f(y)}{y-u} d y .
$$

If we set

$$
G(u)=\left\{\begin{array}{cl}
\frac{a-i}{a+i}, & |u|<1, \\
1, & |u| \geqq 1,
\end{array} \quad \psi(u)=\left\{\begin{array}{cl}
\frac{\phi(u)}{1-i a}, & |u|<1, \\
0, & |u| \geqq 1,
\end{array}\right.\right.
$$

then (7), (10), and (11) imply

$$
F_{+}(u)-G(u) F_{-}(u)=\psi(u) .
$$

Consider the functions $G_{+}(w)$ and $G_{-}(w)$ analytic respectively in $g w>0$ and $g w<0$ and given by

$$
G_{ \pm}(w)=(w+1)^{\beta}(w-1)^{1-\beta},
$$

where the powers are determined by $0<\arg (w \pm 1)<\pi$ in $g w>0$ and $-\pi<\arg (w \pm 1)<0$ in $9 w<0$. The functions $G_{ \pm}$are continuous up to the real axis and we have

$$
\frac{G_{-}(u)}{G_{+}(u)}=G(u), \quad u \neq \pm 1 .
$$

Thus (12) can be written

$$
G_{+}(u) F_{+}(u)-G_{-}(u) F_{-}(u)=G_{+}(u) \psi(u)
$$

and this holds even for $u= \pm 1$. Next define the functions $\Psi_{+}(w)$ and $\Psi_{-}(w)$ analytic in $g w>0$ and $g w<0$ respectively and defined in their respective half-planes by

$$
\Psi_{ \pm}(w)=\frac{1}{2 \pi i} \int_{-1}^{1} \frac{G_{+}(y) \psi(y)}{y-w} d y .
$$

Since $G_{+} \psi$ satisfies a Lipschitz condition, $\Psi_{ \pm}$are continuous up to the real axis and

$$
\Psi_{ \pm}(u)= \pm \frac{1}{2} G_{+}(u) \psi(u)+\frac{1}{2 \pi i} \int_{-1}^{1} \frac{G_{+}(y) \psi(y)}{y-u} d y .
$$

We may now write (13) as 


$$
G_{+}(u) F_{+}(u)-\Psi_{+}(u)=G_{-}(u) F_{-}(u)-\Psi_{-}(u) .
$$

It follows that $G_{+} F_{+}-\Psi_{+}$and $G_{-} F_{-}-\Psi_{-}$are analytic continuations of each other and together represent an entire function. Since this function is bounded it must be a constant $C / 2$. Thus

$$
\begin{aligned}
& F_{+}(u)=\frac{1}{G_{+}(u)}\left[\Psi_{+}(u)+\frac{C}{2}\right], \\
& F_{-}(u)=\frac{1}{G_{-}(u)}\left[\Psi_{-}(u)+\frac{C}{2}\right] .
\end{aligned}
$$

We have now

$$
\begin{aligned}
& \phi(u)=2 F_{+}(u), \\
& \left.f(u)=i \mid F_{-}(u)-F_{+}(u)\right],
\end{aligned}
$$

and from these follow the identities (8) and (9).

The passage to the case of a general $f$ is easy. Let $\left\{f_{n}\right\}$ be a sequence of functions, each twice continuously differentiable and vanishing outside $(-1,1)$, which converges in $L_{2}$ to $f$. We obtain a corresponding sequence $\left\{\phi_{n}\right\}$ which, by the boundedness of the Hilbert transform in $L_{2}$, converges in $L_{2}$ to $\phi$. This shows that the identities involving $\phi_{n}$ and $f_{n}$ imply the corresponding identities involving $f$ and $\phi$.

Note that our assumption $f \in L_{2}$ was largely a matter of convenience. In fact the identities hold assuming merely that $f$ and $\phi$ both belong to $L_{1}$, although the proof in this case makes use of rather deep properties of the Hilbert transform.

4. In our case (see (5) and (6)) $\phi$ is bounded. Since $\gamma( \pm 1)=0$ it follows from (8) that

$$
C=\frac{1}{\pi\left(a^{2}+1\right)^{1 / 2}} \int_{-1}^{1} \frac{\phi(y) \gamma(y)}{ \pm 1-y} d y .
$$

Since $\phi(y)=\operatorname{Pr}\left\{X\left(T_{x}\right) \leqq-1\right\}-\chi(y-x)$ in $(-1,1)$ we have two equations with which to determine $C$ and $\operatorname{Pr}\left\{X\left(T_{x}\right) \leqq-1\right\}$. We obtain

$$
\begin{aligned}
\operatorname{Pr}\left\{X\left(T_{x}\right) \leqq-1\right\} & =\frac{1}{\pi\left(a^{2}+1\right)^{1 / 2}} \int_{x}^{1} \frac{\gamma(y)}{1-y^{2}} d y, \\
C & =-\frac{1}{\pi\left(a^{2}+1\right)^{1 / 2}} \int_{x}^{1} \frac{y \gamma(y)}{1-y^{2}} d y .
\end{aligned}
$$

(Here we use the identities 


$$
\int_{-1}^{1} \frac{\gamma(y)}{1-y^{2}} d y=\pi\left(a^{2}+1\right)^{1 / 2}, \quad \int_{-1}^{1} \frac{y \gamma(y)}{1-y^{2}} d y=0,
$$

both of which follow from

$$
\int_{-1}^{1} \frac{\gamma(y)}{y-u} d y= \begin{cases}\pi\left(a^{2}+1\right)^{1 / 2}\{\operatorname{sgn} u \gamma(u)-u\}, & |u| \geqq 1, \\ \pi\left\{a \gamma(u)-\left(a^{2}+1\right)^{1 / 2} u\right\}, & |u| \leqq 1 .\end{cases}
$$

This in turn follows from

$$
\int_{-\infty}^{\infty} \frac{G_{+}(y)-y}{y-u} d y=\pi i\left\{G_{+}(u)-u\right\}
$$

by taking imaginary parts.)

From (8), (9), (5), and (14) we have

$$
\begin{aligned}
& \operatorname{Pr}\left\{X\left(T_{x}\right) \leqq u\right\}=\chi(u)+\frac{1}{\pi\left(a^{2}+1\right)^{1 / 2}} \int_{x}^{1} \frac{\gamma(y)}{1-y^{2}} d y \\
& -\frac{u^{2}-1}{\pi\left(a^{2}+1\right)^{1 / 2} \gamma(u)} \int_{x}^{1} \frac{\gamma(y)}{\left(1-y^{2}\right)|y-u|} d y, \quad|u| \geqq 1, \\
& K(x, y)=\frac{1-y^{2}}{\pi\left(a^{2}+1\right) \gamma(y)} \int_{x}^{1} \frac{\gamma(u)}{(u-y)\left(1-u^{2}\right)} d u \\
& -\frac{a}{a^{2}+1} \chi(y-x),
\end{aligned}
$$

Nicer looking formulas may be obtained by the following method which, however, requires justification. If we differentiate both sides of (5) and use the fact that $K(x, \pm 1)=0$ we obtain

$$
\frac{d}{d u} \operatorname{Pr}\left\{X\left(T_{x}\right) \leqq u\right\}-\delta(u-x)=\frac{1}{\pi} \int_{-1}^{1} \frac{K_{y}(x, y)}{y-u} d y+a K_{y}(x, u),
$$

where $\delta$ is the Dirac delta function. Thus in equation (7) we have $\phi(u)=-\delta(u-x)$ for $|u|<1$ and $f(y)=K_{y}(x, y)$. The formulas (8) and (9), if they held in this situation, would give

$$
\begin{aligned}
\frac{d}{d u} \operatorname{Pr}\left\{X\left(T_{x}\right) \leqq u\right\} & =\frac{\gamma(x)}{\pi\left(a^{2}+1\right)^{1 / 2} \gamma(u)|x-u|}+\frac{C \operatorname{sgn} u}{\gamma(u)} \\
K_{y}(x, y)= & -\frac{a}{a^{2}+1} \delta(y-x)+\frac{\gamma(x)}{\pi\left(a^{2}+1\right) \gamma(y)(x-y)} \\
& -\frac{C}{\left(a^{2}+1\right)^{1 / 2} \gamma(y)} .
\end{aligned}
$$


We must have $C=0$ since $(d / d u) \operatorname{Pr}\left\{X\left(T_{x}\right) \leqq u\right\}$ is integrable near infinity. Thus

$$
\begin{aligned}
\frac{d}{d u} \operatorname{Pr}\left\{X\left(T_{x}\right) \leqq u\right\}= & \frac{\gamma(x)}{\pi\left(a^{2}+1\right)^{1 / 2} \gamma(u)|x-u|}, \\
K(x, y)= & \frac{\gamma(x)}{\pi\left(a^{2}+1\right)} \int_{-1}^{y} \frac{d u}{\gamma(u)(x-u)} \\
& -\frac{a}{a^{2}+1} \chi(y-x) .
\end{aligned}
$$

We leave as exercises for the reader the proofs of equivalence of (17) with (15) and (18) with (16).

5. As for the moments of $T_{x}$, we have

$$
E\left\{T_{x}^{n}\right\}=n \int_{0}^{\infty} \int_{-1}^{1} t^{n-1} k(x, y, t) d y d t
$$

Now

$$
\begin{aligned}
& k(x, y, t)=\int_{-1}^{1} \cdots \int_{-1}^{1} k\left(x, z_{1}, \tau_{1}\right) k\left(z_{1}, z_{2}, \tau_{2}\right) \cdots \\
& \cdots \\
& \cdots\left(z_{n-2}, z_{n-1}, \tau_{n-1}\right) k\left(z_{n-1}, y, t-\tau_{1}-\cdots-\tau_{n-1}\right) d z_{1} \cdots d z_{n-1}
\end{aligned}
$$

if $\tau_{1}, \cdots, \tau_{n-1} \geqq 0, \sum \tau_{i} \leqq t$. Integration of both sides over this $\tau$ region and then over $t \geqq 0$ gives

$$
\begin{aligned}
\frac{1}{(n-1) !} \int_{0}^{\infty} t^{n-1} k(x, y, t) d t & \\
& =\int_{-1}^{1} \cdots \int_{-1}^{1} K\left(x, z_{1}\right) \cdots K\left(z_{n-1}, y\right) d z_{1} \cdots d z_{n-1} .
\end{aligned}
$$

Therefore

$$
E\left\{T_{x}^{n}\right\}=n ! \int_{-1}^{1} \cdots \int_{-1}^{1} K\left(x, z_{1}\right) \cdots K\left(z_{n-1}, y\right) d z_{1} \cdots d z_{n-1} d y .
$$

The mean first passage time has an especially simple form:

$$
E\left\{T_{x}\right\}=\frac{\gamma(x)}{\left(a^{2}+1\right)^{1 / 2}} .
$$

(We use (18), and a formula similar to (14) but with $1 / \gamma(y)$ instead of $\gamma(y)$ in the integrand.) 
6. One can obtain analogous results in case the unit interval is replaced by an arbitrary finite union of (finite or semi-infinite) intervals. The only complication is that in the analogue of the lemma of $\$ 3$ several constants appear (roughly as many as the number of intervals) and are determined by solving a system of linear equations.

More serious difficulties arise in trying to extend the results to other stable processes. For general symmetric stable processes the two-sided absorption problem was solved independently by several authors $[1 ; 3 ; 6]$. However it seems unlikely that explicit formulas may be obtained for asymmetric stable processes with exponent $\alpha \neq 1$, 2. For $\alpha<1$, for example, the analogue of equation (6) is

$$
\begin{aligned}
\operatorname{Pr}\left\{X\left(T_{x}\right)\right. & \leqq-1\}-\chi(u-x) \\
& =C(\alpha) \int_{-1}^{1} \frac{K(x, y) \operatorname{sgn}(y-u)}{|y-u|^{\alpha}} d y+a K(x, u), \quad|u| \leqq 1,
\end{aligned}
$$

where $C(\alpha)$ is a constant depending on $\alpha$. From this equation it is possible to express $K(x, y)$ in terms of the eigenvalues and eigenfunctions of the kernel $|y-u|^{-\alpha} \operatorname{sgn}(y-u)$ on $(-1,1)$, but nothing much simpler can be expected.

\section{REFERENCES}

1. R. M. Blumenthal, R. K. Getoor and D. B. Ray, On the distribution of first hits for the symmetric stable processes, Trans. Amer. Math. Soc. vol. 99 (1961) pp. 540-554.

2. M. Kac and H. Pollard, The distribution of the maximum of partial sums of independent random variables, Canad. J. Math. vol. 2 (1950) pp. 375-384.

3. H. Kesten, Random walks with absorbing barriers and Toeplitz forms, Illinois J. Math. vol. 5 (1961) pp. 267-290.

4. F. Spitzer, Some theorems concerning 2-dimensional Brownian motion, Trans. Amer. Math. Soc. vol. 87 (1958) pp. 187-197.

5. F. G. Tricomi, Integral equations, New York, Interscience, 1957.

6. H. Widom, Stable processes and integral equations, Trans. Amer. Math. Soc. vol. 98 (1961) pp. 430-449.

Cornell University 